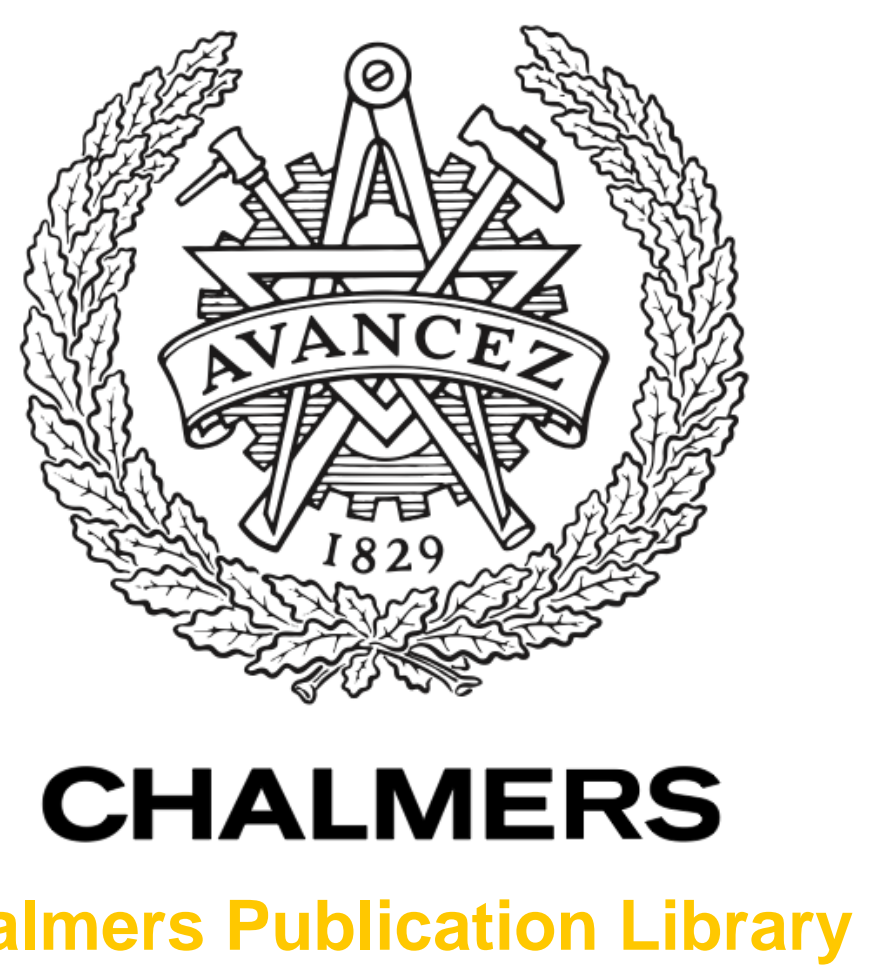

Challmers Publication Library

\title{
Carbon nanotube based bolometer
}

This document has been downloaded from Chalmers Publication Library (CPL). It is the author's version of a work that was accepted for publication in:

JETP Letters (ISSN: 0021-3640)

Citation for the published paper:

Tarasov, M. ; Svensson, J. ; Kuzmin, L. (2006) "Carbon nanotube based bolometer". JETP Letters, vol. 84(5), pp. 267-270.

http://dx.doi.org/10.1134/S0021364006170085

Downloaded from: http://publications.lib.chalmers.se/publication/25777

Notice: Changes introduced as a result of publishing processes such as copy-editing and formatting may not be reflected in this document. For a definitive version of this work, please refer to the published source. Please note that access to the published version might require a subscription. 


\title{
Bolometer Based on Carbon Nanotubes
}

\author{
M. Tarasov ${ }^{a, b}$, J. Svensson $^{c}$, J. Weis $^{b}$, L. Kuz'min ${ }^{a, b}$, and E. Campbell ${ }^{c}$ \\ ${ }^{a}$ Institute of Radio Engineering and Electronics, Russian Academy of Sciences, \\ ul. Mokhovaya 17/7, Moscow, 125009 Russia \\ e-mail:tarasov@hitech.cplire.ru \\ ${ }^{b}$ Chalmers University of Technology, SE41296 Gothenburg, Sweden \\ ${ }^{c}$ Gothenburg University, SE41296 Gothenburg, Sweden \\ Received June 28, 2006; in final form, July 26, 2006
}

\begin{abstract}
The contacts of single carbon nanotubes and bundles of carbon nanotubes with the superconducting and metallic electrodes are investigated in order to create bolometers and electron coolers. The tunneling contacts of the carbon nanotubes with aluminum electrodes are obtained. The current-voltage characteristics of junctions are analyzed for temperatures from room temperature to $300 \mathrm{mK}$. The resistance of individual nanotubes is primarily determined by defects and is too large for applications. The use of the bundles of carbon nanotubes makes it possible to considerably reduce the resistance of the bolometer, which is determined by a small number of conducting tubes with good tunneling contacts with the electrodes. The energy gap is equal to hundreds and ten of millivolt in the former and latter cases, respectively. Structures containing the bundles of carbon nanotubes can be described in a model with a Schottky barrier. The samples with the bundles of carbon nanotubes exhibit the bolometric response to external high-frequency radiation at a frequency of $110 \mathrm{GHz}$ with an amplitude up to $100 \mu \mathrm{V}$ and a temperature voltage response to $0.4 \mathrm{mV} / \mathrm{K}$.
\end{abstract}

PACS numbers: 74.50.+r, 85.25.Pb

DOI: $10.1134 / \mathrm{S} 0021364006170085$

As previously shown theoretically, a strong bolometric effect and effective electron cooling are possible in bolometric structures with the superconductor-insulator-normal metal (SIN) junctions [1]. The electron cooling from 300 to $100 \mathrm{mK}$ was experimentally obtained [2]. The limiting characteristics of the proposed devices can be estimated using the relation $P=$ $\Sigma v\left(T_{e}^{5}-T_{o}^{5}\right)$ for the energy transfer from electrons to phonons or the relation $T_{e}=\left(T_{o}^{5}+P / \Sigma v\right)^{1 / 5}$ for the electron temperature, where $\Sigma$ is the constant of the material, $v$ is the absorber volume, and $T_{o}$ is the phonon temperature. According to these expressions, in order to increase the efficiency of the radiation-induced heating of the absorber and to improve the electron cooling below the lattice temperature, it is necessary to strongly reduce the absorber volume. In an ordinary bolometer with metallic thin-film absorber manufactured using electron lithography [3], the sizes are $10 \times 0.2 \times$ $0.05 \mu \mathrm{m}$, whereas the sizes of carbon nanotubes of the same length are $10 \times 0.001 \times 0.001 \mu \mathrm{m}$; i.e., the volume in the latter case is smaller by four orders of magnitude.

It is commonly accepted that single-wall carbon nanotubes have diameters $1-4 \mathrm{~nm}$ and consist of a graphene sheet rolled into a cylinder. In dependence on the bond structures, the conductivity of a nanotube can be semiconducting, metallic, or superconducting [4]. Many-wall nanotubes can have diameters up to $50 \mathrm{~nm}$ and be superconducting at temperatures below $12 \mathrm{~K}$.
The existing technologies make it possible to grow single nanotubes between electrodes on the substrate or to deposit prepared carbon nanotubes on the substrate between the electrodes by varying the electric field. A separate problem is the formation of the electric contacts with the nanotubes. If a carbon nanotube is a onedimensional object, the resistance at the interface with the three-dimensional electrode cannot be lower than the quantum resistance $R_{Q}=h / 2 e^{2}=12.9 \mathrm{k} \Omega$ per one mode [5]. Since electrons have two spin orientations, the lowest possible resistance of the contact with the one-dimensional conductor is no less than $6.5 \mathrm{k} \Omega$ [6].

\section{PRODUCTION OF THE SAMPLES}

In our work, the carbon nanotubes are produced by the chemical vapor deposition method and plasmaenhanced chemical vapor deposition method [7-9] using catalytic synthesis from the gas phase by the hydrogenation of acetylene on iron, nickel, or niobium nuclei at a temperature of $900^{\circ} \mathrm{C}$ at atmospheric pressure. The second method is applied to form the nanotubes between sharp electrodes directly on the substrate. After deposition or formation on the substrate, superconducting aluminum electrodes are deposited on carbon nanotubes. This method allows the production of the carbon nanotubes including metallic tubes.

The photomasks are developed and produced for both methods of producing the carbon nanotubes. A 


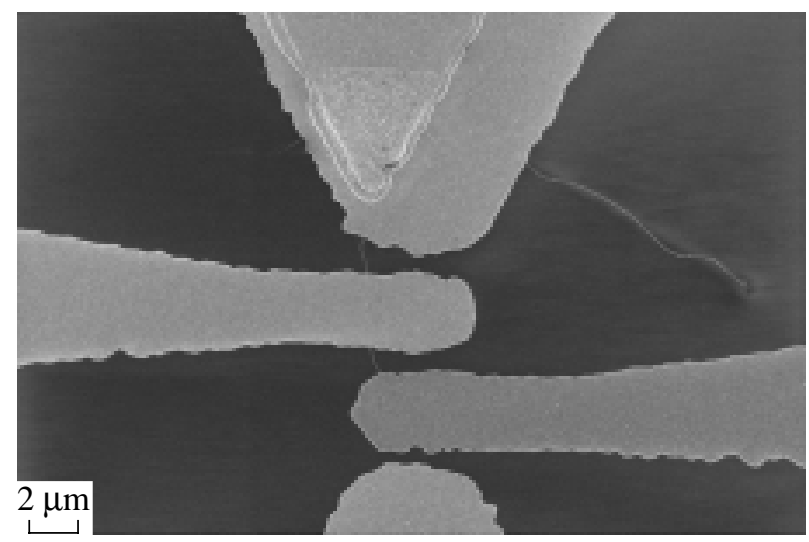

Fig. 1. Two nanotubes grown on the silicon substrate and covered with aluminum electrodes.

niobium film is deposited as a lower layer with sharp growing points between which nanotubes are either grown or electrically aligned (see Fig. 1). In the latter case, small droplets of the solution containing nanotubes with a mean diameter of about $1.5 \mathrm{~nm}$ are deposited between electrodes. An ac voltage with an amplitude of about $2 \mathrm{~V} / \mu \mathrm{m}$ is applied to the electrodes for $3-$ $10 \mathrm{~s}$. Thus, under the action of the dielectrophorese mechanism, the nanotubes attract to the substrate and are located in the gap between the electrodes, where they are joined to the substrate by the van der Waals forces. Single nanotubes and bundles of nanotubes can be deposited in dependence on the process parameters. Up to one third of tubes in bundles with thousands of tubes can be semimetallic with a narrow gap and up to one twentieth of them can be completely metallic. The dielectrophorese process is used to control and accelerate the process of bundling nanotubes. The alternating voltage leads to the polarization of the nanotubes. The tubes in the static field would be located along the field lines in the directions to the electrodes. Since the field is inhomogeneous, forces from the different sides are different and tubes aligned along the field direction execute forward-backward oscillations in the alternating field. Owing to these motions, the dipole moment interacts with the field and generates forces downwards to the substrate.

Two or four contacts with the tube are formed at the second stage by depositing the aluminum electrodes or three-layer electrodes consisting of a $0.5-\mathrm{nm}$ titanium film, 5-nm palladium film, and 100-nm aluminum film. After extraction from the sputtering setup, the aluminum surface at the interface with the carbon nanotubes can oxidize and form a tunneling barrier. For the case of palladium, the Schottky barrier can be formed at the interface between metal and a semiconducting or semimetallic tube. In this case, titanium was used to improve adhesion and palladium is used owing to good wettability with carbon nanotubes.
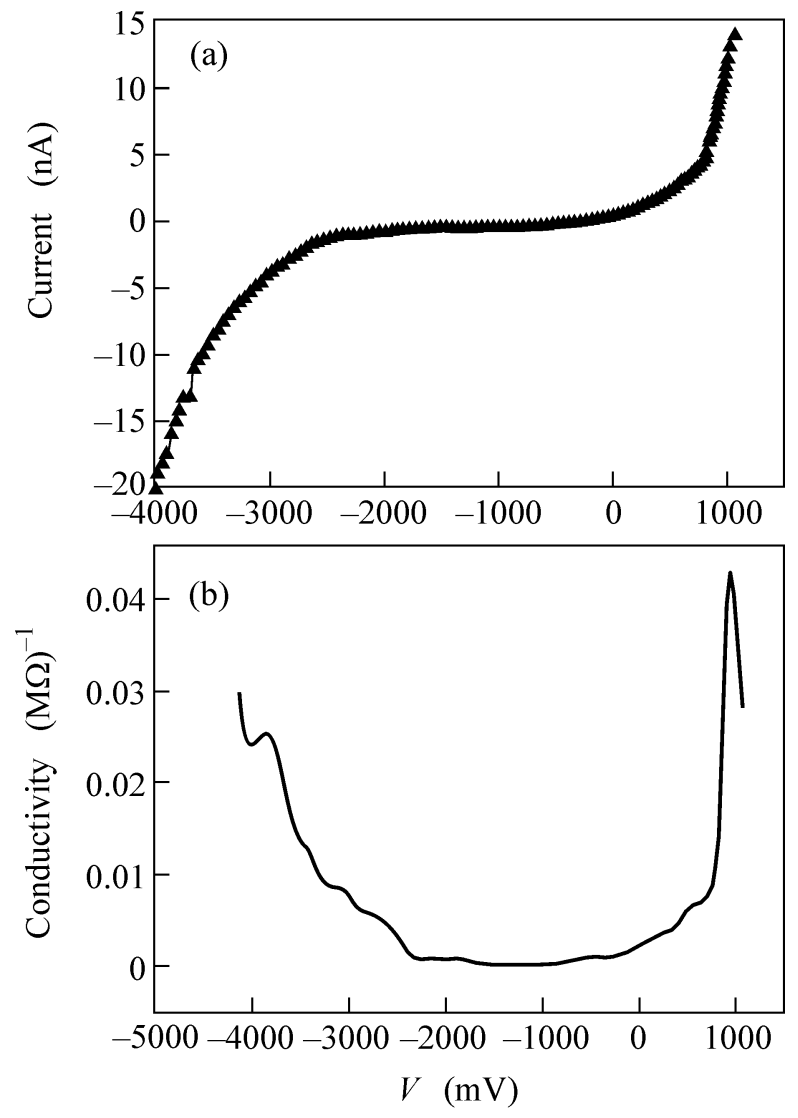

Fig. 2. (a) Current-voltage characteristic and (b) differential conductivity of the Al-AlOx-carbon nanotube tunneling junction as measured by the three-probe scheme at a temperature of $176 \mathrm{~K}$.

\section{EXPERIMENT}

The current-voltage characteristics of produced junctions with single nanotubes (see Fig. 1) are shown in Fig. 2. When the three-probe method is used for measurements, the current-voltage characteristics exhibit two energy barriers, one of them is located near $2 \mathrm{eV}$ and corresponds to aluminum, whereas the other is located near $0.5 \mathrm{eV}$ and corresponds to carbon. The produced nanotubes contain many defects and, for this reason, their resistance is very high; in this case, tunneling conduction through one-electron mechanism and Coulomb blockade occur at a temperature of about $300 \mathrm{mK}$.

The bundles of carbon nanotubes are examined in order to reduce the resistance of the bolometers owing to the high conductivity of metallic nanotubes with a small number of defects in the next series of the experiments. In this case, the resistance is determined by the tubes with the lowest resistance and their number is comparatively small, because the fraction of metallic tubes is usually equal to several percent. The photograph and current-voltage characteristic of one of these samples of nanotubes with four aluminum electrodes 
are shown in Figs. 3 and 4, respectively. The resistance of such a structure at a temperature of $300 \mathrm{mK}$ is equal to several kiloohms compared to the Gigaohm resistance of single tubes at the same temperature. Sections that are asymmetric with respect to zero and have low dynamic resistance exist for small biases. The existence of these sections can be naturally explained in the semiconductor junction model. In the presence of many parallel tubes, one part of them can be represented in the equivalent scheme as back-to-back diodes, whereas another part, as antiparallel connection diodes. An important quantity is the scale of this nonlinearity and a possible energy gap or barrier that is on the order of hundreds of millivolt for single, assumingly semiconducting, carbon nanotubes and on the order of ten millivolts for bundles in which metallic and semimetallic tubes prevail. For various temperatures, such nonlinearity can be described as a diode with the Schottky barrier (semiconductor-normal metal) or super-Schottky diode (superconductor-semiconductor).

We measured the voltage response of the bundle of the carbon nanotubes irradiated by high-frequency radiation. The signal source at a frequency of $110 \mathrm{GHz}$ is a generator based on an avalanche diode with an output power of about $10 \mathrm{~mW}$. A signal is weakened in an ECCOSORB absorber, is modulated by a mechanical modulator, and is fed through an optical window with cold IR filters on the sample containing the bundle of nanotubes. The substrate with the sample is mounted on the flat surface of the elongated hyperhemispherical silicon lens $12.6 \mathrm{~mm}$ in diameter. According to our estimates, the signal power absorbed by the bolometer is much lower than $1 \mathrm{nW}$. A constant bias current is applied to the sample and voltage response is measured on the synchronous detector. Figure 5 shows the response and current-voltage characteristic measured at a temperature of $4.2 \mathrm{~K}$. The maximum output signal reaches $100 \mu \mathrm{V}$. The current-voltage characteristic and response of the carbon nanotubes of the bolometer are qualitatively similar to the current-voltage characteristic and response, respectively, of a usual superconductor-insulator-normal metal-insulator-superconductor (SINIS) bolometer with a normal-metal strip as an absorber [3], but with a wide energy gap.

The temperature dependences of the voltage on the same sample are measured at a given current of $0.3 \mu \mathrm{K}$ (Fig. 6). The value $d V / d T=0.4 \mathrm{mV} / \mathrm{K}$ is close to the temperature response for metallic thin-film SINIS bolometers [3].

\section{DISCUSSION OF THE RESULTS}

To explain the results, it is convenient to use the nonlinearity model such as a diode with the Schottky barrier (DSB). The current-voltage characteristic of the DSB can be represented as $I=I_{s}\left[\exp \left(e V / \eta k_{b} T\right)-1\right]$, where $I$ is the current through the barrier, $V$ is the voltage, $I_{s}$ is the saturation current, $e$ is the elementary

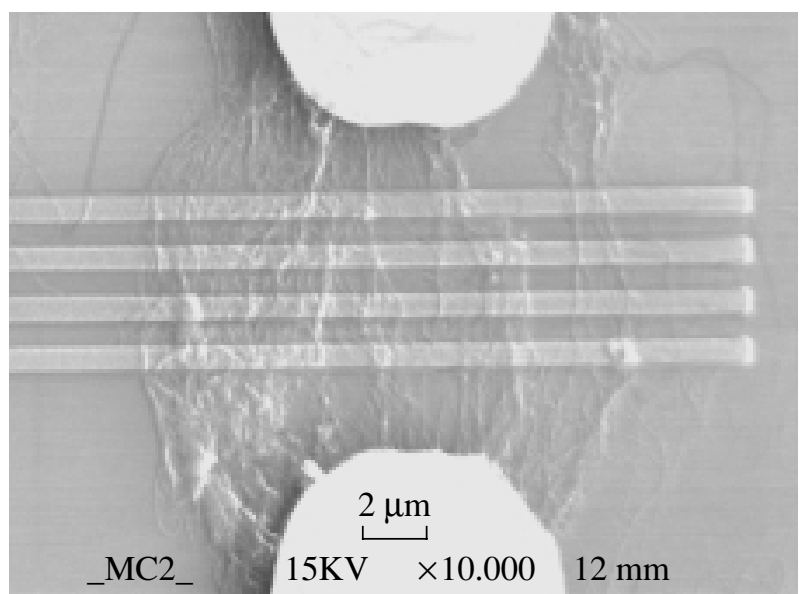

Fig. 3. Image of the bundle of nanotubes with four aluminum electrodes.

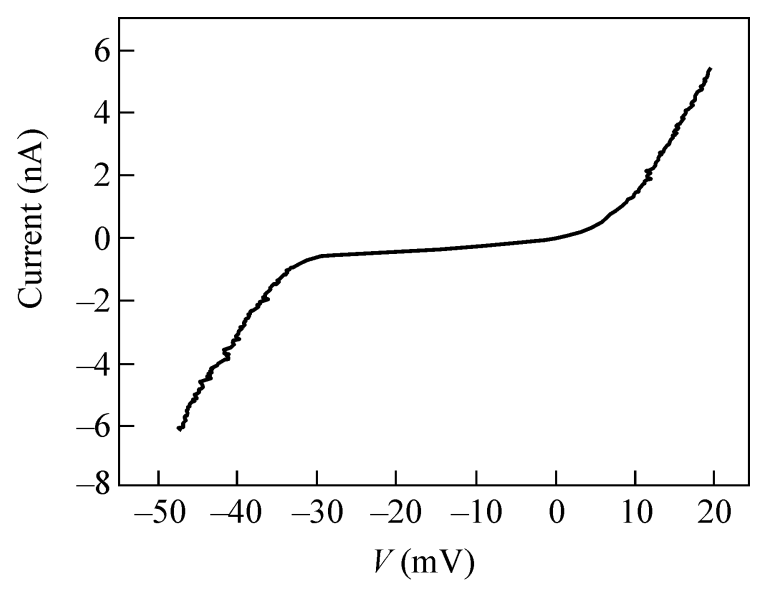

Fig. 4. Current-voltage characteristic of the bundle of nanotubes as measured in the two-probe scheme at a temperature of $300 \mathrm{mK}$

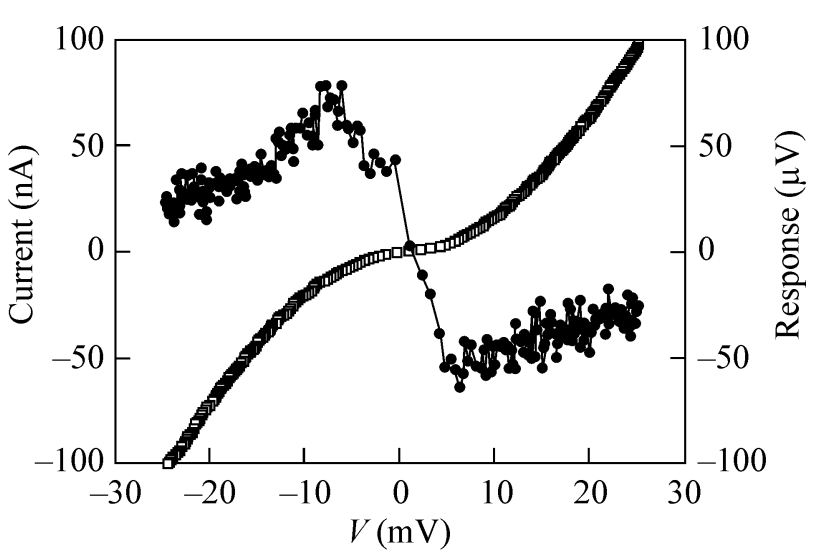

Fig. 5. Current-voltage characteristic of the bundle of carbon nanotubes and voltage response to $110-\mathrm{GHz}$ radiation as measured at a temperature of $T=4.2 \mathrm{~K}$.

\section{JETP LETTERS Vol. $84 \quad$ No. 52006}




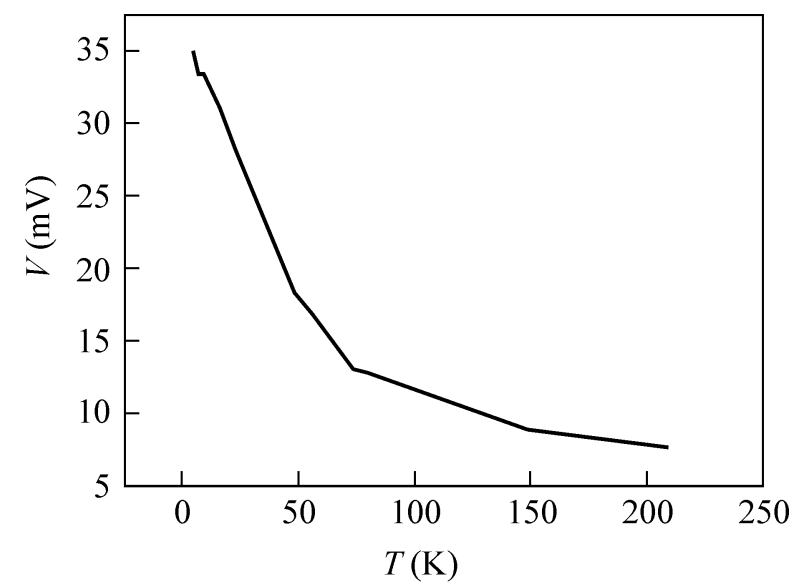

Fig. 6. Temperature dependence of the voltage on the sample at a given current of $0.3 \mu \mathrm{A}$.

charge, $T$ is the temperature, $k_{\mathrm{B}}$ is the Boltzmann constant, and $\eta$ is the junction imperfection factor. An important parameter of the DSB is its cutoff frequency, which is determined by the leakage resistance and capacitance. For current DSBs, this quantity can exceed a plasma frequency of $20 \mathrm{THz}$ [10]. For weakly doped diodes, a good detector response can be observed up to $30 \mathrm{THz}$ [11]. The noise temperature of a mixer based on the DSB can decrease to $3500 \mathrm{~K}$ for $2.5 \mathrm{THz}$ [12]. The basic factor limiting the characteristics of DSB mixers is the distributed resistance, which is proportional to the junction diameter and can be extremely small in our case of the carbon nanotubes. It is worth mentioned that electron cooling can be realized in a superconductorsemiconductor structure [13]. If the production technology is optimized, such effect can also be observed in samples with the bundles of carbon nanotubes. The super-Schottky diodes can be mentioned as direct detectors of radiation with a noise-equivalent power of $5 \times 10^{-16} \mathrm{~W} / \mathrm{Hz}^{1 / 2}$ and as a mixer with a noise temperature of about $6 \mathrm{~K}$, as shown in [14]. The combination of both effects, electron cooling and bolometric response, can be expected in the bundles of carbon nanotubes. One of the advantages of the DSB is the absence of the necessity of the formation of an additional tunnel barrier, because the Schottky barrier is automatically formed at the interface between the semiconductor and normal metal. As a corroboration of our model, we point to recent works on investigation [15] of Schottky diodes with carbon nanotubes and on experimental investigation [16] of the bolometric photoresponse in a suspended film consisting of one-wall nanotubes.

This work was supported by the Swedish Institute, the Russian Foundation for Basic Research (project no. 05-02-19650), and the Russian Federal Agency for Science and Innovation (contract no. 02.434.11.1010).

\section{REFERENCES}

1. L. Kuzmin, I. Devyatov, and D. Golubev, Proc. SPIE 3465, 193 (1998).

2. L. Kuzmin, I. Agulo, M. Fominsky, et al., Supercond. Sci. Technol. 17, 400 (2004).

3. M. Tarasov, M. Fominsky, A. Kalabukhov, and L. Kuzmin, JETP Lett. 76, 507 (2002).

4. I. Takesue, J. Haruyama, N. Kobayashi, et al., Phys. Rev. Lett. 96, 057001 (2006).

5. Y. Imry and R. Landauer, Rev. Mod. Phys. 71, 2 (1999).

6. K. S. Yngvesson, Appl. Phys. Lett. 87, 043503 (2005).

7. http://www.fy.chalmers.se/atom/research/nanotubes/ production.xml.

8. S. Dittmer, J. Svensson, and E. Campbell, Curr. Appl. Phys. 4, 595 (2004).

9. R. E. Morjan, M. S. Kabir, S. W. Lee, et al., Curr. Appl. Phys. 4, 591 (2004).

10. K. S. Champlin and G. Eisenstein, IEEE Trans. Microwave Theory Tech. 26, 31 (1978).

11. D. Tsang and S. Schwartz, Appl. Phys. Lett. 30, 263 (1977).

12. M. Gaidis, H. Pickett, C. Smith, et al., IEEE Trans. Microwave Theory Tech. 48, 733 (2000).

13. A. Savin, M. Prunnila, P. P. Kivinen, et al., Appl. Phys. Lett. 79, 1471 (2001).

14. F. Vernon, M. Millea, M. Bottjer, et al., IEEE Trans. Microwave Theory Tech. 25, 286 (1977).

15. M. Yang, K. Teo, W. Milne, and D. Hasko, Appl. Phys. Lett. 87, 253116 (2005).

16. M. Itkis, F. Borondics, A. Yu, and R. Haddon, Science 312, 413 (2006).

Translated by R. Tyapaev 